\title{
Assessment of incidental findings in 232 whole-exome sequences from the Baylor-Hopkins Center for Mendelian Genomics
}

\author{
Julie Jurgens, BSc${ }^{1}$, Hua Ling, $\mathrm{PhD}^{2}$, Kurt Hetrick, MS², Elizabeth Pugh, $\mathrm{PhD}^{2}$, \\ Francois Schiettecatte, $\mathrm{PhD}^{3}$, Kimberly Doheny, $\mathrm{PhD}^{2}$, Ada Hamosh, MD, $\mathrm{MPH}^{1}$, \\ Dimitri Avramopoulos, MD, PhD ${ }^{1}$, David Valle, $\mathrm{MD}^{1}$ and Nara Sobreira, MD, $\mathrm{PhD}^{1}$
}

\begin{abstract}
Purpose: In March 2013 the American College of Medical Genetics and Genomics published a list of 56 genes with the recommendation that pathogenic and likely pathogenic variants detected incidentally by clinical sequencing be reported to patients. As an initial step in determining the practical consequences of this recommendation in the research setting, we searched for variants in these genes in 232 whole-exome sequences from the Baylor-Hopkins Center for Mendelian Genomics.
\end{abstract}

Methods: We identified rare, nonsynonymous, and splicing singlenucleotide variants and insertions/deletions and assessed variant classification using the Human Gene Mutation, Emory, and ClinVar databases. We analyzed the burden of mutation in each of the 56 genes and determined which variants should be reported to patients.

Results: Our filtering resulted in 249 distinct variants, with a mean of 1.69 variants per individual. Half of these were novel missense mutations not classified by any of the three reference databases. Of 101 variants listed in the Human Gene Mutation Database, 48 were also in ClinVar and 3 were also in Emory; half of these shared variants were classified discordantly between databases. Some genes consistently had greater variation than others. In total, $0.86 \%$ of individuals had a reportable incidental variant.

Conclusion: These observations demonstrate some current challenges of assessing phenotypic consequences of incidental variants for counseling patients.

Genet Med advance online publication 8 January 2015

Key Words: American College of Medical Genetics and Genomics recommendations; incidental findings; secondary findings; variant classification; whole-exome sequencing

\section{INTRODUCTION}

Incidental findings in whole-exome and whole-genome sequencing are variants of known or possible pathology identified in genes unrelated to the initial reason for which sequencing tests were ordered. Because large-scale sequencing is increasingly used in a clinical setting, standardizing procedures for identifying, classifying, and reporting variants is of great importance. In 2013 the American College of Medical Genetics and Genomics (ACMG) released a recommendation suggesting that known pathogenic or expected pathogenic constitutional variants detected in 56 genes, representing 24 conditions with well-known guidelines for prevention or treatment, should be reported to patients sequenced in a clinical setting. ${ }^{1}$ Shortly after the release of these recommendations, the Clinical Sequencing Exploratory Research Consortium and Electronic Medical Records and Genomics Network followed by releasing a set of guidelines for the return of incidental findings within genomics research studies. ${ }^{2}$ These guidelines recommended that medically actionable incidental variants be returned to willing research participants but exempted researchers from an obligation to search for these variants.

Although incidental variants are unexpected, when variant pathogenicity is certain, the information enables patients to make proactive decisions about their health and inform their physician. However, some variants are of uncertain significance, or the initial interpretation of variant pathogenicity may be subject to change. ${ }^{3}$ Reporting these variants of uncertain significance to patients may lead to misinterpretation of the results, produce unnecessary anxiety, and diminish confidence in genetic test results in general. Thus, transmittal of these results requires accurate and thoughtful genetic counseling and careful evaluation of available sources of variant classification to maximize benefits and minimize harm to patients.

At the Baylor-Hopkins Center for Mendelian Genomics (http://mendeliangenomics.org), ${ }^{4}$ we utilize whole-exome sequencing to detect variants responsible for Mendelian disorders with unknown molecular bases. Because we sequence across the exome, we capture not only variants that are responsible for the patient phenotype but also incidental variants that

${ }^{1}$ McKusick-Nathans Institute of Genetic Medicine, Johns Hopkins University School of Medicine, Baltimore, Maryland, USA; ${ }^{2}$ Center for Inherited Disease Research, McKusick-Nathans Institute of Genetic Medicine, Johns Hopkins University School of Medicine, Baltimore, Maryland, USA; ${ }^{3}$ FS Consulting, Salem, Massachusetts, USA. Correspondence: Nara Sobreira (nsobrei2@jhmi.edu) 
may predispose to and/or cause other unrelated disorders. Returning meaningful results to research participants through their physicians is a goal of our center, so understanding the clinical significance of incidental findings detected at the research level and knowing which of these should be returned to patients is of special interest.

Although the ACMG guidelines for reporting incidental findings are geared toward a clinical setting, they provide a well-defined, targeted set of genes from among the many interrogated in research sequencing. Thus our results on this set may inform policies for handling incidental findings. Here we examine the implementation of the ACMG clinical guidelines within a research setting by assessing the spectrum of rare, functional variation in the 56 genes in whole-exome sequences of 232 individuals in 89 families sequenced at the Baylor-Hopkins Center for Mendelian Genomics for a variety of Mendelian disorders with unknown molecular bases, some of which have features that overlap with the disorders covered by the ACMG list. We then assess variant interpretation by evaluating classification and reportability according to available databases and approaches taken by others, as described in the literature.

\section{MATERIALS AND METHODS}

All research participants were counseled regarding the possible outcomes of whole-exome sequencing and signed a consent form approved by the Johns Hopkins University School of Medicine Institutional Review Board. Participants chose whether they would like to receive information regarding primary or incidental variants found during the course of this study and were given the opportunity to opt out or in at any time.

Whole-exome sequences from 232 individuals in 89 families sequenced at Johns Hopkins University were analyzed for rare splicing or nonsynonymous single-nucleotide variants (SNVs) and insertions/deletions (indels) in the 56 reportable genes from the ACMG guidelines using the analysis feature of the Web-based system PhenoDB. ${ }^{5}$

Briefly, whole-exome sequencing was done on the Illumina HiSeq2000 platform (Illumina, San Diego, CA) using pairedend, 75- or 100-bp reads. Genomic DNA (51 Mb) comprising consensus coding sequence exons and adjacent intron sequences was captured with the Agilent SureSelect Human All Exon V4 51Mb Kit (Agilent Technologies, Santa Clara, CA). FASTQ files were aligned to the reference genome (GRCh37; Ensembl core database release 50_361) ${ }^{6}$ with the Burrows-Wheeler Alignment (BWA 0.5.10) tool, ${ }^{7}$ resulting in SAM/BAM output. ${ }^{8}$ Polymerase chain reaction duplicates were flagged with Picard. ${ }^{9}$ Local realignment around indels, base call quality score recalibration, and reduced-read BAMs were performed with GATK $2.3-9 .{ }^{10}$ Multisample SNV and indel calling was performed on the reduced-read BAM files with GATK's UnifiedGenotyper. Variant sites were filtered with GATK's Variant Quality Score Recalibration best practices, ${ }^{11}$ and heterozygous genotypes were excluded if they did not have at least five alternate allele reads. Sequencing coverage of the 56 genes is listed in Supplementary Table S1 online.

We selected for nonsynonymous SNVs, exonic indels, and splice-junction variants (two base pair sequences at the $5^{\prime}$ and $3^{\prime}$ ends of each intron) in each of the 56 reportable genes. Nonsynonymous SNVs and exonic variants were defined by RefSeq Gene coordinates. ${ }^{12,13}$ Next, we filtered these variants on the basis of their minor allele frequencies (MAFs), with exclusion of variants with a MAF $\geq 0.01$ in the 1000 Genomes Project (April 2012 release), ${ }^{6}$ Exome Variant Server (http://evs. gs.washington.edu/EVS/), ${ }^{14} \mathrm{dbSNP}$ build $137,{ }^{15,16}$ and our inhouse control database (CIDRVar $51 \mathrm{Mb}$ ), which includes data from 50 individuals sequenced in-house with the Agilent $51 \mathrm{Mb}$ exome capture kit (Agilent Technologies).

Next, we analyzed the variants for prior classification in the Human Gene Mutation Database (HGMD), ${ }^{17,18}$ ClinVar, ${ }^{19,20}$ and the Emory Genetics Laboratory Variant Classification Catalog. ${ }^{21,22}$ We then defined the burden of variants in each individual sequenced, the mutability of each gene (the number of rare functional variants per gene and the number of rare functional variants per kilobase of coding region), and the classification and reportability of each variant.

\section{RESULTS}

In the 232 exomes we identified 249 distinct variants in the 56-gene ACMG target set, some of which occurred in more than one individual, for a total of 391 variants. A total of 124 variants were shared between family members, and 18 variants were shared between unrelated individuals. There was an average of 1.69 variants per individual, with a range of $0-7$ variants per individual. There were only 45 people (19.4\%) with no variants, and in the 232 exomes we found at least one variant in 45 of the 56 genes (80.4\%; Supplementary Table S1 online).

We then stratified these 249 distinct variants by type (Table 1). Most variants were missense (231/249, or 92.8\%). There were also four nonsynonymous/splice variants (1.6\%), two splice variants $(0.8 \%)$, three frameshifting indels $(1.2 \%)$, five nonframeshifting indels (2.0\%), and four nonsense variants (1.6\%).

Next, we checked the classification of these variants in three databases: HGMD, ClinVar, and Emory. Of the 249 distinct variants identified, $126(50.6 \%)$ were classified by at least one of the three databases: $54(42.8 \%)$ were represented solely in

Table 1 Classification of the 249 variants by mutation type

\begin{tabular}{lc} 
Variant type & Variants $(\boldsymbol{n})$ \\
\hline Missense single-nucleotide variant & 231 \\
Nonframeshifting insertion/deletion & 5 \\
Nonsense & 4 \\
Exonic/splicing & 4 \\
\hline Frameshifting insertion/deletion & 3 \\
Splicing & 2 \\
\hline Total & 249 \\
\hline
\end{tabular}




\section{ORIGINAL RESEARCH ARTICLE}

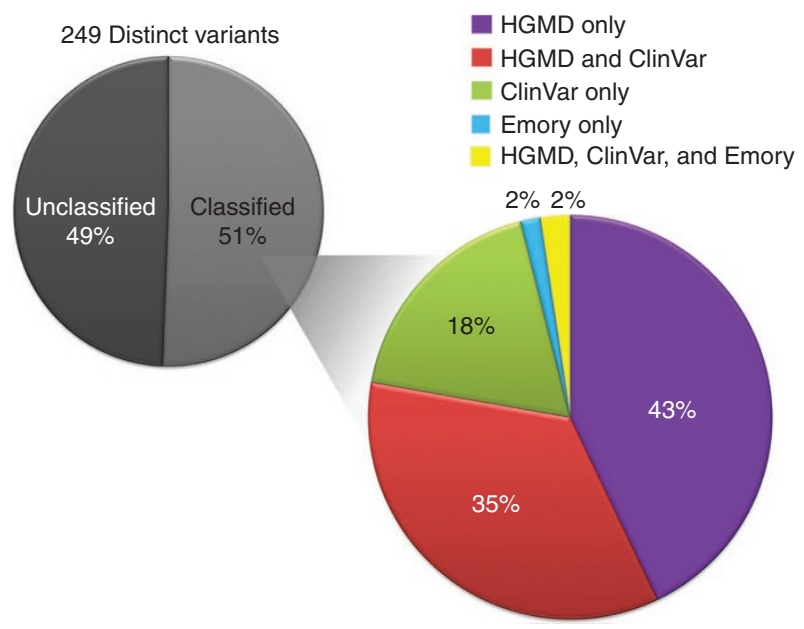

Figure 1 Classification of the 249 variants by the Human Gene Mutation Database (HGMD), ClinVar, and Emory databases.

HGMD; 44 (34.9\%) were in HGMD and ClinVar; 23 (18.3\%) were in ClinVar alone; 2 (1.6\%) were in Emory alone; and another $3(2.4 \%)$ were in all three databases (Figure 1).

In total, $101 / 249$ variants (40.5\%) were listed in HGMD (94 missense SNVs (93.1\%), 2 nonsynonymous exonic/splicing variants (2.0\%), 2 splicing variants (2.0\%), 1 nonsense SNV (1.0\%), and 2 nonframeshifting indels $(2.0 \%)$ ). More importantly, HGMD classified $74(72.8 \%)$ as disease-causing mutations, 24 $(24.3 \%)$ as possible disease-causing mutations, $1(1.0 \%)$ as a functional polymorphism, and $2(1.9 \%)$ as disease-associated polymorphisms reported to have a significant association with disease $(P<0.05)$, along with some evidence of functionality.

Of the 148 variants not classified by HGMD, 23 variants in 13 genes were described by ClinVar and 2 variants in 2 genes were listed in Emory. This left a total of 123/249 variants (49.4\%) that were not present in any database (Figure 1).

The classification of variants within these three databases was often discordant. Of the 101 variants represented in HGMD, 48 were also in ClinVar. Three of these 48 variants were also in Emory. Almost half of these shared variants (22/48 variants, or $45.8 \%)$ were given discordant classifications among databases (Supplementary Table S2 online).

Next, we examined the effects of gene size and evolutionary constraint on the number of functional variants within each gene in a manner similar to that described by Petrovski et al..$^{23}$ Some genes consistently had greater variation than others. For instance, BRCA2, APOB, CACNA1S, and DSP had the greatest number of variants per gene (Supplementary Table $\mathbf{S 1}$ online). Because increased coding length increases the target size for mutation, larger genes are expected to have a higher number of variants than are smaller genes. To explore this possibility, we analyzed the number of variants per gene against the size (kilobases) of the consensus coding sequence of the gene. We found that the number of variants per gene generally increased with the size of coding sequence, and the number of variants showed a $62.8 \%$ correlation with the size of the consensus coding sequence (Figure 2).

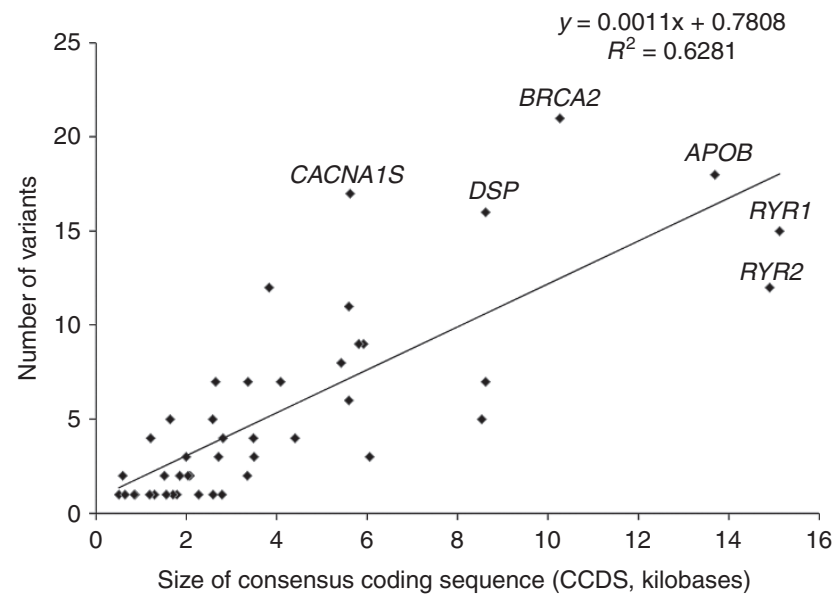

Figure 2 The number of variants per gene plotted against the size (kilobases) of the consensus coding sequence of the gene.

We also found that some genes (e.g., RB1, PMS2, and MLH1) consistently have a lower variant density (variants per kilobase coding sequence) than others (e.g., MYBPC3, TMEM43, and MYL3) (Supplementary Table S1 online). The values of variant density range from 0.4 variants $/ \mathrm{kb}$ in $R B 1$ to 3.4 variants $/ \mathrm{kb}$ in MYL3. As described by others, a possible explanation for this variation in mutational burden, despite correction for coding sequence length, is varying degrees of evolutionary constraint. ${ }^{23}$ Certain genes are under a higher degree of purifying selection than others, and this is likely reflected by less variation per unit coding length.

\section{Determining reportable variants}

To determine which variants should be reported to our research participants, we adopted a stringent set of measures outlined in a recent paper by Dorschner et al. ${ }^{24}$ Their criteria incorporate various lines of evidence in assessing variant pathogenicity, including comparison between variant minor allele frequencies and incidences of the corresponding disorders; familial segregation data; observation in unrelated affected individuals; de novo events in a trio; and protein truncation in disorders caused by haploinsufficiency. If a variant is classified as pathogenic or likely pathogenic by these criteria, it is considered reportable to the patient.

We applied these criteria to all 391 variants in the 56 ACMG genes detected in 232 exomes. In total, we found two pathogenic variants (in $M S H 2$ and $M Y L K$ ) and three likely pathogenic variants (in LMNA, MYBPC3, and MUTYH), as shown in Table 2. One of these genes, MUTYH, is responsible for autosomal recessive conditions (adenomas, multiple colorectal, FAP type 2 (OMIM 608456); colorectal adenomatous polyposis, autosomal recessive, with pilomatricomas (OMIM 132600)). ${ }^{25}$ Because current ACMG guidelines recommend reporting only homozygous variants in $M U T Y H,{ }^{1}$ we would not report this heterozygous variant to the patient. Because the patients in our cohort underwent whole-exome sequencing to explain a variety of potential Mendelian disorders, some of which have overlapping features with the disorders covered by the ACMG 


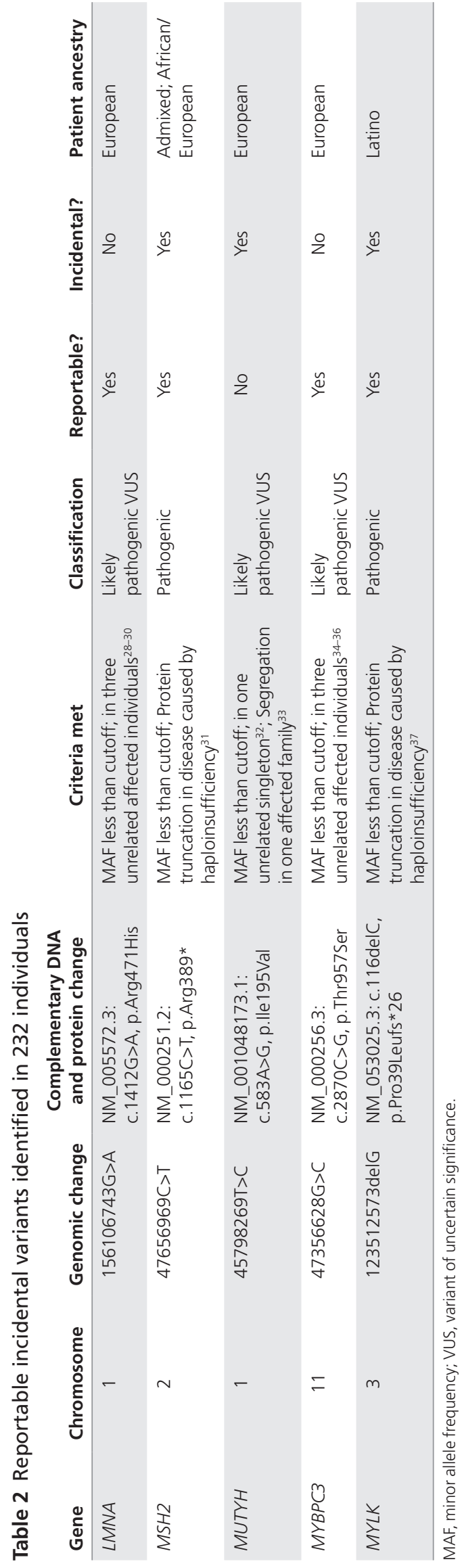

list, we recognized that our cohort may have an enrichment of reportable variants as compared with the general population. Indeed, two patients, one with a likely pathogenic variant in $L M N A$ and one with a likely pathogenic variant in $M Y B P C 3$, had clinical phenotypes that included dilated cardiomyopathy, so we did not count these two cases toward our final number of incidental variants. In total, this means that 2 of 232 individuals, or $0.86 \%$ of our sample, had a reportable incidental variant in one of the $56 \mathrm{ACMG}$ genes.

\section{DISCUSSION}

In June 2014 Jarvik et al. ${ }^{2}$ released recommendations for dealing with incidental variants in the research arena. They suggested that researchers be exempted from an obligation to search for variants outside the intended scope of their study but that actionable variants be reported to research participants when discovered. The present study describes the workflow for identification and classification of incidental findings in the Johns Hopkins component of the Baylor-Hopkins Center for Mendelian Genomics based on the ACMG recommendations and on the classification criteria described by Dorschner et al. ${ }^{24}$

In 232 exomes we identified a total of 391 rare $(\mathrm{MAF}<1 \%)$ variants in the $56 \mathrm{ACMG}$ genes (1.69 variants/individual), 249 of which were distinct variants. Most of these variants were missense (231/249, or $92.8 \%$ ), and half were not classified by any of the three databases (HGMD, ClinVar, and Emory) that we used to assess variant classification (123/249 variants, or $49.4 \%$ ). Because these novel variants were not represented in the variant databases, they could not be used for comparative analyses of classifications among the three databases.

Next we used the criteria described by Dorschner et al. ${ }^{24}$ to classify the 391 total variants identified in 232 exomes and found that $2 / 232$ individuals, or $0.86 \%$ of our sample, had an incidental reportable variant in one of the 56 ACMG genes. Overall, this analysis was quite time consuming, but we were able to automate parts of it, such as filtering variants whose frequency was higher than that predicted by the frequency of their associated disorders. This step reduced the number of distinct variants from 249 (obtained in our initial analyses using a $1 \%$ MAF cutoff) to 163 , so only $65.5 \%$ of the original variants required further assessment. We were also able to automate selection of variants in the HGMD, ClinVar, and Emory classification databases using our Web-based system, PhenoDB. ${ }^{5}$ Although we did not consider the classifications from these databases in support of variant pathogenicity or reportability, the databases provided useful citations from the literature and clinical observations for some variants. This information reduced the time required for manual review. Protein truncation was automatically predicted but required manual inspection to confirm that protein truncation is an established cause of the disorder associated with the respective gene. The most time-consuming part of the process was reviewing the literature for each variant to count familial segregations, de novo events, and singleton reports. This step required additional expertise regarding diagnoses 
of the disorders on the ACMG list. Moreover, evaluation of cases in the literature was somewhat subjective.

Several other studies have analyzed reportable incidental findings within whole-exome or whole-genome sequences to date. The study by Dorschner et al., ${ }^{24}$ whose criteria we adopted for our analysis of reportable variants, found that 14/1,000 individuals sequenced (1.4\%) had a pathogenic or likely pathogenic reportable variant in one of the 52 ACMG genes responsible for adult-onset conditions. Johnston et al. ${ }^{26}$ found that $8 / 572$ (1.39\%) individuals sequenced through the ClinSeq project had a reportable variant in one of 37 cancer genes; 23 of these are on the ACMG list. These numbers are similar to ours and to those in the original ACMG guidelines, which anticipated that $1 \%$ of individuals sequenced would have a reportable variant in one of these 56 genes. ${ }^{1}$

Another recent report by Lawrence et al. ${ }^{27}$ detected 27/543 individuals $(\sim 5 \%)$ sequenced through the National Institutes of Health Undiagnosed Diseases Program with an incidental variant in one of the 56 ACMG genes. Their method differed from ours in a few ways, which may have contributed to the discrepancy in the frequency of incidental findings. For instance, they used reported functional studies as evidence of pathogenicity, required fewer informative meioses to count familial cosegregation events with disease, did not apply MAF cutoffs, and used designations in variant classification databases as evidence in support of variant pathogenicity. In addition, a few individuals in their cohort had a phenotype related to the reported incidental finding, and some incidental variants were repeated due to familial transmission. These differences in methodology may have contributed to the discrepancies in the numbers of reportable incidental findings.

During this process we recognized that identifying and classifying the multitude of incidental variants that arise from next-generation sequencing is a time-consuming and subjective process dependent on the particular methodologies of each individual investigator. We confirmed this last observation when we found that almost half (22/48, or 45.8\%) of the 101 variants represented in at least two databases were given discordant classifications between them. We compared these database classifications with our variant classifications made based on the method developed by Dorschner et al. ${ }^{24}$ and found that variant classifications from the ClinVar and Emory databases, but not the HGMD database, were generally consistent with our variant classifications (Supplementary Table S2 online). This is likely because the HGMD aims to provide a comprehensive listing of all reported variants and draws its classifications from reports in the literature ${ }^{17,18}$ that may not be accurate, consistent, up to date, or sufficient to provide support of variant pathogenicity. For these reasons, HGMD is generally less conservative than the other databases.

The abundance of rare and novel missense variants in our cohort made accurate assessment of variant pathogenicity more challenging, because all novel and many rare missense variants must initially be classified as variants of unknown significance. For many rare variants, familial segregation data or de novo events in a trio were not available or sufficient to define variants as pathogenic or likely pathogenic.

When we observed de novo variants in a trio or cosegregation of a variant with disease, family information could be used as evidence supporting pathogenicity and reportability. Although failure of a variant to segregate might be considered evidence against pathogenicity, we did not use this information in our pathogenicity evaluations because many disorders on the ACMG list are adult-onset conditions and could manifest late in unaffected family members. Family sequence data also are useful for determining the phase of multiple heterozygous variants in a single gene and thus can be used to find compound heterozygous variants. When individuals rather than families are sequenced, classifying variants as pathogenic or likely pathogenic may be more difficult because these sources of information are not available. Support for pathogenicity can be obtained in other ways, however, such as literature searches. Thus family information is helpful but not necessary for determining variant pathogenicity in most cases.

We found that prediction is even more difficult for genes that are subject to more variation because of their increased size or tolerance for variation. Because functional information was not available for most variants, 152 of 249 distinct variants (61.0\%) were classified as variants of uncertain significance.

Some variants had been previously described in the dbSNP, Exome Variant Server, and/or 1000 Genomes databases, and of 249 distinct variants, $126(50.6 \%)$ were classified by at least one of the three databases used to assess variant classification (HGMD, ClinVar, and/or Emory). The available classifications in these cases were helpful but not always clear, mainly because of discrepancies among bioinformatic prediction databases or functional studies, low numbers of unrelated affected individuals with the same variant, misreporting of variant pathogenicity in the literature or classification databases, or occurrence of variants in large or highly mutable genes. These inconsistencies all point to the need for a single, centralized database and methodology for variant classification.

Our study has several limitations. First, many individuals in our cohort were ascertained for various Mendelian disorders. Because of this, two of the reportable findings could not be considered incidental, in that they may actually have contributed to the patients' phenotype. In addition, our cohort was relatively small, and $73 \%$ of individuals self-identified as being of European descent. Moreover, we may have missed some variants because of low coverage in some regions of the 56 ACMG genes. Another limitation was the MAF cutoffs that we used. For our early comparisons of variant classification between databases, we used a $1 \%$ MAF cutoff, which may be too high for some disorders and necessitate more time for manual review of variants. On the other hand, our later assessments of variant reportability used more stringent MAF cutoffs based on expected disease prevalence. This increased stringency may have resulted in the exclusion of variants that were in fact pathogenic. In addition, the 
method we used to find reportable variants fails to consider functional studies. Because of this, we may have excluded some variants that have been functionally characterized as pathogenic or included variants that show milder or less conclusive functional consequences.

An alternative sequencing method such as whole-genome sequencing would increase the detection of incidental findings as well as variants of uncertain significance because of the increased coverage of this method and the challenge of interpreting noncoding variants. Whole-genome sequencing will uncover structural variants and copy-number variants as well. The ACMG recommendations currently apply only to SNVs and indels, however, so this additional variation-some of which may be significant-would not be considered under the current guidelines. ${ }^{1}$

Based on this experience, we suggest that clinicians and researchers interested in returning incidental variants found by next-generation sequencing adopt a uniform, well-defined set of criteria for variant classification. Here we applied the ACMG recommendations and the classification criteria defined by Dorschner et al. ${ }^{24}$ but we suggest that the addition of bioinformatic prediction tools, genic intolerance scores, and functional studies to the criteria would make variant classification more complete. We also expect that research and clinical laboratories performing nextgeneration sequencing will soon be more engaged in submitting variants to manually curated classification databases, improving the interpretation of variants and counseling of patients.

\section{SUPPLEMENTARY MATERIAL}

Supplementary material is linked to the online version of the paper at http://www.nature.com/gim

\section{ACKNOWLEDGMENTS}

The authors thank all of our research participants, who made this study possible. J.J. is a trainee of the Predoctoral Training Program in Human Genetics at Johns Hopkins University and is supported by National Institutes of Health grant T32GM07814. This work was funded by a grant from the National Human Genome Research Institute (U54HG006542).

\section{DISCLOSURE}

The authors declare no conflict of interest.

\section{REFERENCES}

1. Green RC, Berg JS, Grody WW, et al.; American College of Medical Genetics and Genomics. ACMG recommendations for reporting of incidental findings in clinical exome and genome sequencing. Genet Med 2013;15:565-574.

2. Jarvik GP, Amendola LM, Berg JS, et al.; eMERGE Act-ROR Committee and CERC Committee; CSER Act-ROR Working Group. Return of genomic results to research participants: the floor, the ceiling, and the choices in between. Am J Hum Genet 2014;94:818-826.

3. Bell CJ, Dinwiddie DL, Miller NA, et al. Carrier testing for severe childhood recessive diseases by next-generation sequencing. Sci Trans/ Med 2011;3:65ra4.

4. Baylor-Hopkins Center for Mendelian Genomics. http:// mendeliangenomics.org.

5. Hamosh A, Sobreira N, Hoover-Fong J, et al. PhenoDB: a new web-based tool for the collection, storage, and analysis of phenotypic features. Hum Mutat 2013;34:566-571.
6. The 1000 Genomes Project Consortium. An integrated map of genetic variation from 1,092 human genomes. Nature 2012;491: 56-65.

7. Li H, Durbin R. Fast and accurate short read alignment with Burrows-Wheeler transform. Bioinformatics 2009;25:1754-1760.

8. Li H, Handsaker B, Wysoker A, et al.; 1000 Genome Project Data Processing Subgroup. The Sequence Alignment/Map format and SAMtools. Bioinformatics 2009;25:2078-2079.

9. Picard Software. http://picard.sourceforge.net. Accessed June-August 2013.

10. McKenna A, Hanna M, Banks E, et al. The Genome Analysis Toolkit: a MapReduce framework for analyzing next-generation DNA sequencing data. Genome Res 2010;20:1297-1303.

11. DePristo MA, Banks E, Poplin R, et al. A framework for variation discovery and genotyping using next-generation DNA sequencing data. Nat Genet 2011;43:491-498.

12. Pruitt KD, Tatusova T, Maglott DR. NCBI Reference Sequence (RefSeq): a curated non-redundant sequence database of genomes, transcripts and proteins. Nucleic Acids Res 2005;33(Database issue):D501-D504.

13. RefSeq Genes Track, The Cancer Genome Workbench. https://cgwb.nci. nih.gov/cgi-bin/hgTrackUi?hgsid=79902\&c=chr21\&g=refGene. Accessed 25 April 2012.

14. Exome Variant Server. Seattle: NHLBI GO Exome Sequencing Project (ESP), http://evs.gs.washington.edu/EVS/. Accessed June-August 2013.

15. Sherry ST, Ward MH, Kholodov M, et al. dbSNP: the NCBI database of genetic variation. Nucleic Acids Res 2001;29:308-311.

16. Database of Single Nucleotide Polymorphisms. Bethesda, MD: National Center for Biotechnology Information, National Library of Medicine. (dbSNP Build 137). http://www.ncbi.nlm.nih.gov/SNP/. Accessed June-August 2013.

17. Stenson PD, Ball EV, Mort M, et al. Human Gene Mutation Database (HGMD): 2003 update. Hum Mutat 2003:21:577-581.

18. The Human Gene Mutation Database. http://www.hgmd.cf.ac.uk/ad/. Accessed April 2013.

19. Landrum MJ, Lee JM, Riley GR, et al. ClinVar: public archive of relationships among sequence variation and human phenotype. Nucleic Acids Res 2014;42(Database issue):D980-D985.

20. ClinVar Variant Database. http://www.ncbi.nlm.nih.gov/clinvar/. Accessed June-August 2013.

21. Bean LJ, Tinker SW, da Silva C, Hegde MR. Free the data: one laboratory's approach to knowledge-based genomic variant classification and preparation for EMR integration of genomic data. Hum Mutat 2013;34: 1183-1188.

22. Emory Genetics Laboratory. http://genetics.emory.edu/egl/emvclass/emvclass. php. Accessed June-August 2013.

23. Petrovski S, Wang Q, Heinzen EL, Allen AS, Goldstein DB. Genic intolerance to functional variation and the interpretation of personal genomes. PLoS Genet 2013;9:e1003709.

24. Dorschner MO, Amendola LM, Turner EH, et al.; National Heart, Lung, and Blood Institute Grand Opportunity Exome Sequencing Project. Actionable, pathogenic incidental findings in 1,000 participants' exomes. Am J Hum Genet 2013;93:631-640.

25. Online Mendelian Inheritance in Man, $\mathrm{OMIM}{ }^{\circledR}$. McKusick-Nathans Institute of Genetic Medicine, Johns Hopkins University: Baltimore, MD, 2014. http:// omim.org/. Accessed June-August 2013.

26. Johnston JJ, Rubinstein WS, Facio FM, et al. Secondary variants in individuals undergoing exome sequencing: screening of 572 individuals identifies highpenetrance mutations in cancer-susceptibility genes. Am J Hum Genet 2012;91:97-108.

27. Lawrence $L$, Sincan $M$, Markello $T$, et al. The implications of familial incidental findings from exome sequencing: the NIH Undiagnosed Diseases Program experience. Genet Med 2014;16:741-750.

28. Astejada MN, Goto K, Nagano A, et al. Emerinopathy and laminopathy clinical, pathological and molecular features of muscular dystrophy with nuclear envelopathy in Japan. Acta Myol 2007;26:159-164.

29. Parks SB, Kushner JD, Nauman D, et al. Lamin A/C mutation analysis in a cohort of 324 unrelated patients with idiopathic or familial dilated cardiomyopathy. Am Heart J 2008;156:161-169.

30. Cowan J, Li D, Gonzalez-Quintana J, Morales A, Hershberger RE. Morphological analysis of 13 LMNA variants identified in a cohort of 324 unrelated patients with idiopathic or familial dilated cardiomyopathy. Circ Cardiovasc Genet 2010;3:6-14.

31. Payne SR, Kemp CJ. Tumor suppressor genetics. Carcinogenesis 2005;26:2031-2045. 
32. Sieber OM, Lipton L, Crabtree M, et al. Multiple colorectal adenomas, classic adenomatous polyposis, and germ-line mutations in MYH. N Engl J Med 2003;348:791-799.

33. Morak M, Massdorf T, Sykora H, Kerscher M, Holinski-Feder E. First evidence for digenic inheritance in hereditary colorectal cancer by mutations in the base excision repair genes. Eur J Cancer 2011;47:1046-1055.

34. Ehlermann P, Weichenhan D, Zehelein J, et al. Adverse events in families with hypertrophic or dilated cardiomyopathy and mutations in the MYBPC 3 gene. BMC Med Genet 2008;9:95.
35. Rodríguez-García MI, Monserrat L, Ortiz M, et al. Screening mutations in myosin binding protein $\mathrm{C} 3$ gene in a cohort of patients with hypertrophic cardiomyopathy. BMC Med Genet 2010;11:67.

36. Olivotto I, Girolami F, Ackerman MJ, et al. Myofilament protein gene mutation screening and outcome of patients with hypertrophic cardiomyopathy. Mayo Clin Proc 2008;83:630-638.

37. Wang L, Guo DC, Cao J, et al. Mutations in myosin light chain kinase cause familial aortic dissections. Am J Hum Genet 2010;87:701707. 